\title{
Management of psychosis and schizophrenia in adults: summary of updated NICE guidance
}

The authors of this Practice article (BMJ 2014;348:g1173, doi:10.1136/bmj.g1173) would like to clarify a recommendation made under the "Care across all phases-physical health" section. The second bullet point in this section advises readers to "Be aware of the potential impact of reducing nicotine on the metabolism of other drugs, particularly clozapine and

olanzapine." However, the authors have since been advised that it may cause confusion to say "reducing nicotine" in this context because it is the hydrocarbons in cigarette smoke that induce the metabolism of clozapine (and in some cases olanzapine). To avoid any potential misunderstanding, this sentence should have read: "Be aware of the potential significant impact of reducing cigarette smoking on the metabolism of other drugs, particularly clozapine and olanzapine."

Cite this as: BMJ 2014;348:g2234

๑) BMJ Publishing Group Ltd 2014 